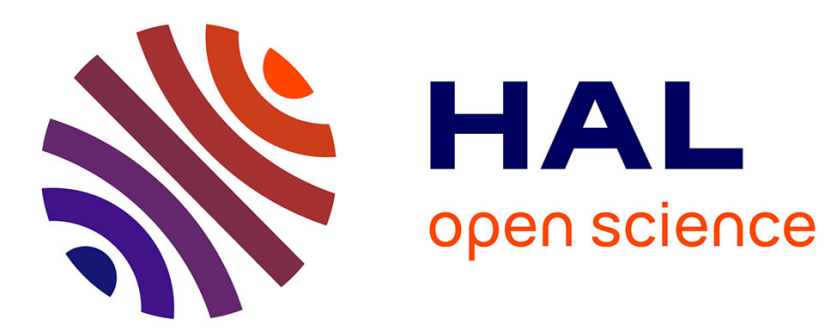

\title{
Travelling waves in the Fermi-Pasta-Ulam lattice
}

Gérard Iooss

\section{To cite this version:}

Gérard Iooss. Travelling waves in the Fermi-Pasta-Ulam lattice. Nonlinearity, 2000, 13, pp.18. 10.1088/0951-7715/13/3/319. hal-01271021

\section{HAL Id: hal-01271021 \\ https://hal.univ-cotedazur.fr/hal-01271021}

Submitted on 9 Feb 2016

HAL is a multi-disciplinary open access archive for the deposit and dissemination of scientific research documents, whether they are published or not. The documents may come from teaching and research institutions in France or abroad, or from public or private research centers.
L'archive ouverte pluridisciplinaire HAL, est destinée au dépôt et à la diffusion de documents scientifiques de niveau recherche, publiés ou non, émanant des établissements d'enseignement et de recherche français ou étrangers, des laboratoires publics ou privés. 


\title{
Travelling waves in the Fermi-Pasta Ulam lattice
}

\author{
Gérard Iooss \\ Institut Universitaire de France, \\ INLN, UMR CNRS-UNSA 6618 \\ 1361 route des Lucioles \\ F-06560 Valbonne \\ e.mail: iooss@inln.cnrs.fr
}

\begin{abstract}
We consider travelling wave solutions on a one-dimensional lattice, corresponding to mass particles interacting nonlinearly with their nearest neighbor (Fermi-Pasta-Ulam model). A constructive method is given, for obtaining all small bounded travelling waves for generic potentials, near the first critical value of the velocity. They all are solutions of a finite $d i$ mensional reversible $O D E$. In particular, near (above) the first critical velocity of the waves, we construct the solitary waves whose global existence was proved by Friesecke et Wattis [1], using a variational approach. In addition, we find other travelling waves like (i) superposition of a periodic oscillation with a non zero averaged stretching or compression between particules, (ii) mainly localized waves which tend to uniformly stretched or compressed lattice at infinity, (iii) heteroclinic solutions connecting a stretched pattern with a compressed one.
\end{abstract}

\section{Introduction and Formulation of the problem}

We consider the dynamics of the classical one-dimentional lattice given by

$$
\ddot{X}_{n}=V^{\prime}\left(X_{n+1}-X_{n}\right)-V^{\prime}\left(X_{n}-X_{n-1}\right), n \in \mathbb{Z}
$$

where $X_{n}(\widetilde{t}), \tilde{t} \in \mathbb{R}$, gives the position of the $n t h$ particle, $V$ is the potential due to nearest-neighbor interaction. We are interested in travelling waves solutions of (1). The system (1) has a special physical importance, mainly due to its apparent simplicity, and to the discovery from Fermi, Pasta, Ulam [1] about the (numerically found) time behavior of solutions with sinusoidal initial condition, having recurrence properties, meaning that it does not mix different modes, despite of its a prori non integrability. Important results on its localized solutions are given by Friesecke \&Wattis in [2], using a variational approach. We refer the reader to references in [2] for numerical results on localized waves in one dimensional lattices. In the present work, we follow the lines of the method initiated by Iooss and Kirchgässner [4] on a similar system. With the ansatz 
$X_{n}(\widetilde{t})=\widetilde{x}(n \tau-\widetilde{t})$, after scaling the time as $\widetilde{t}=\tau t$, and denoting $x(t)=\widetilde{x}(\tau t)$, system (1) is transformed to

$$
\ddot{x}(t)=\tau^{2}\left(V^{\prime}[x(t+1)-x(t)]-V^{\prime}[x(t)-x(t-1)]\right)
$$

which is a scalar "neutral" or "advance-delay" differential equation. We refer to the basic paper [4] for general references on advance-delay differential equations, related to our type of problem (second order differential). However, we give a constructive original method for obtaining the "small" solutions of (2) for velocities of the waves close to the first critical value (called the "sound velocity" in [2]). We show that they all belong to a finite dimensional center manifold, and are given by the small bounded solutions of an ordinary differential equation. This reduction result follows from the work [4] on a similar problem, which was inspired by the analogous reduction available for elliptic systems in strips (see the seminal work [6] of Kirchgässner ). We do not reproduce here the complete proof of this reduction process, refering the reader to [4] for details. The additional difficulty here is the invariance property of our system under addition of a constant, which leads to a systematic double zero eigenvalue for the linearized operator, for all values of the parameters (velocity of the waves, curvature at 0 of the potential). We solve this difficulty and concentrate on the extensive study of all types of small bounded solutions of (2). Our results are summed up in 3 theorems, valid for generic potentials. The first one gives the localized solutions, and is the result analogous to the one of theorem 1 in [2]. The second theorem gives "mainly localized" solutions, which are asymptotic to a stretched or a compressed lattice ("stretched" means that $X_{n+1}-X_{n}$ is enlarged, "compressed" means the opposite). Depending on the local properties of the potential, we also show the existence of an "heteroclinic" solution connecting a stretched lattice with a compressed one. The last theorem gives large families of periodic solutions $(\dot{x}(t)$ is time periodic), where the pattern has time periodic oscillations around a stretched or a compressed uniform state (this averaged state may be the basic uniform state $(x=0)$.

The reversibility symetry given by $x(t) \longmapsto-x(-t)$ plays a capital role in what follows. An important remark is that this system is invariant under the transformation $x \longmapsto x+q$ for any $q \in \mathbb{R}$. Moreover we have to keep in mind that there is a family of "trivial" solutions given by $x(t)=a t+b$ for any $a$ and $b$ in $\mathbb{R}$. They correspond to uniformly stretched or compressed patterns in (1).

In what follows, we need to specify the behavior of $V^{\prime}$ near 0 :

$$
V^{\prime}(x)=\alpha x+\beta x^{2}+\gamma x^{3}+\delta x^{4}+\ldots
$$

the essential assumption being that

$$
V^{\prime \prime}(0)=\alpha>0 .
$$

For obtaining non trivial results, we also need that, at least one of the higher order coefficients of the Taylor expansion of $V^{\prime}$ at the origin is non zero. We shall concentrate our analysis to the cases when $\beta$ or $\gamma$ are non zero. 
We are now ready to look at this system with the eyes of the work [4].

Instead of treating (2) directly, we introduce a new variable $v \in[-1,1]$ and functions $X(t, v)=x(t+v)$. The notation $U(t)(v)=(x(t), \xi(t), X(t, v))^{T}$ indicates our intention to construct $U$ as a map from $\mathbb{R}$ into some function space living on the $v$-interval $[-1,1]$. We use the notations $\xi(t)=\dot{x}(t), \delta^{1} X(t, v)=$ $X(t, 1)$, and $\delta^{-1} X(t, v)=X(t,-1)$. Equation (2) can now be written as follows

$$
\partial_{t} U=L_{\mu} U+M_{\tau}(U),
$$

where $\mu=\alpha \tau^{2}$, and $L_{\mu}$ is the linear, nonlocal operator

$$
L_{\mu}=\left(\begin{array}{ccc}
0 & 1 & 0 \\
-2 \mu & 0 & \mu\left(\delta^{1}+\delta^{-1}\right) \\
0 & 0 & \partial_{v}
\end{array}\right)
$$

and

$$
M_{\tau}(U)=\tau^{2}\left(0, g\left(\delta^{1} X-x\right)-g\left(x-\delta^{-1} X\right), 0\right)^{T}
$$

where we define $g(x)=V^{\prime}(x)-\alpha x=O\left(x^{2}\right)$ as $x \rightarrow 0$. Moreover, we require that $X(t, 0)=x(t)$.

As in [4], we introduce Banach-spaces $\mathbb{H}$ and $\mathbb{D}$ for $U(v)=(x, \xi, X(v))^{T}$

$$
\begin{aligned}
& \mathbb{H}=\mathbb{R}^{2} \times\left(C^{0}[-1,1]\right) \\
& \mathbb{D}=\left\{U \in \mathbb{R}^{2} \times\left(C^{1}[-1,1]\right) / X(0)=x\right\}
\end{aligned}
$$

with the usual maximum norms. The operator $L_{\mu}$ then maps $\mathbb{D}$ into $\mathbb{H}$ continuously. The nonlinearity $M_{\tau}$ is supposed to satisfy $M_{\tau} \in C^{k}(\mathbb{D}, \mathbb{D}), k \geq 1$, and

$$
\left\|M_{\tau}(U)\right\|_{\mathbb{D}} \leq c(\rho)\|U\|_{\mathbb{D}}^{2}
$$

for all $U \in \mathbb{D}$ with $\|U\|_{\mathbb{D}} \leq \rho$; $\rho$ being an arbitrary positive constant. In our particular case $V^{\prime}$ and $g \in C^{2}(\Omega)$ suffices for the validity of the assumption on $M_{\tau} ; \Omega$ denotes an open neighborhood of $0 \in \mathbb{R}$.

The operator $L_{\mu}$, acting in $\mathbb{H}$ with domain $\mathbb{D}$, has a compact resolvent in $\mathbb{H}$. Moreover, $L_{\mu}$ and $M_{\tau}$, both anticommute with the reflexion $S$ in $\mathbb{H}$, given by

$$
S(x, \xi, X)^{T}=(-x, \xi,-X \circ s)^{T},
$$

where $X \circ s(v)=X(-v)$. Therefore, (3) is reversible.

\section{The spectrum of $L_{\mu}$ and its resolvent}

To determine the spectrum $\sum \equiv \sum L_{\mu}$ of $L_{\mu}$, the resolvent equation

$$
\left(\lambda \mathbb{I}-L_{\mu}\right) U=F
$$

has to be solved for any given $F=\left(f_{0}, f_{1}, F_{2}\right)^{T} \in \mathbb{H}$, with $\lambda \in \mathbb{C}$, and $U=$ $(x, \xi, X)^{T} \in \mathbb{D}$. This is possible provided that $N(\lambda ; \mu) \neq 0$, where

$$
N(\lambda ; \mu)=-\lambda^{2}+2 \mu(\cosh \lambda-1) .
$$


Indeed, we obtain

$$
\begin{aligned}
x & =-[N(\lambda ; \mu)]^{-1}\left(\lambda f_{0}+f_{1}+\mu \widetilde{f}_{\lambda}\right), \\
\xi & =-[N(\lambda ; \mu)]^{-1}\left\{\left[\lambda^{2}+N(\lambda ; \mu)\right] f_{0}+\lambda f_{1}+\mu \lambda \widetilde{f}_{\lambda}\right\}, \\
X(v) & =e^{\lambda v} x-\int_{0}^{v} e^{\lambda(v-s)} F_{2}(s) d s,
\end{aligned}
$$

with

$$
\widetilde{f}_{\lambda}=\int_{0}^{1}\left[-e^{\lambda(1-s)} F_{2}(s)+e^{-\lambda(1-s)} F_{2}(-s)\right] d s .
$$

Since $N(\lambda ; \mu)$ is an entire function of $\lambda$ for every $\mu \in \mathbb{R}$, the spectrum $\sum L_{\mu}$ consists of isolated eigenvalues $\lambda$. They are roots of $N(\lambda ; \mu)$, and thus have finite multiplicities.

Remark, that $L_{\mu}$ is real and that $S L_{\mu}+L_{\mu} S=0$ holds. $\sum L_{\mu}$ is then invariant under $\lambda \mapsto \bar{\lambda}$ and $\lambda \mapsto-\lambda$. Thus, $\sum L_{\mu}$ is invariant under reflexion on the real - and the imaginary axis in $\mathbb{C}$. Thus, we can restrict the following considerations to $\lambda=p+i q$ with nonnegative $p$ and $q$.

The central part $\sum_{0} \equiv \sum_{0} L_{\mu}=\sum L_{\mu} \cap i \mathbb{R}$ of the spectrum is determined by $N(i q ; \mu)=0, q \in \mathbb{R}$, i.e.

$$
q^{2}+2 \mu(\cos q-1)=0 .
$$

Using the same type of proof as in [4] (quite elementary here, with the function $\left.q^{-2}(1-\cos q)\right)$, we have the following

Lemma 1 (i) For each $\mu>0$, there exists $p_{0}>0$, such that all $\lambda \in \sum L_{\mu} \backslash \sum_{0}$ satisfy $|\operatorname{Re} \lambda| \geq p_{0}$.

(ii) Let $\lambda=p+i q \in \sum$ then

$$
|q| \leq 2 \sqrt{\mu+4 e^{-2}} \cosh (p / 2)
$$

holds.

(iii) For $0<\mu<1,0$ is the only eigenvalue on the imaginary axis. It has multiplicity two. There are only two real eigenvalues $\pm \lambda$, moreover these eigenvalues tend towards 0 as $\mu \rightarrow 1$. For $\mu \geq 1$ the eigenvalue 0 is the only one on the real axis.

(iv) For $\mu=1$, the eigenvalue 0 is quadruple, with a $4 \times 4$ Jordan block.

(v) There is an increasing unbounded sequence $\mu_{n}, n=0,1,2, \ldots$ of (critical) values of the parameter $\mu$ (see figure 1), such that $\mu_{0}=1$ and for $\mu=\mu_{n}, n>$ 1 , there is one pair $\pm i q_{n}$ of double non semi-simple imaginary eigenvalues in addition to the double non semi-simple eigenvalue at 0 , and $2 n-1$ pairs of simple imaginary eigenvalues $\pm i q_{j}^{\prime}$, such that $0<q_{j}^{\prime}<q_{n}$ (see figure 1 ).

Since the bifurcations in system (2) will only occur when the cardinality of $\sum_{0} L_{\mu}$ changes, the first relevant case occurs for $\mu$ near 1 , which means that the velocity $1 / \tau$ of these bifurcating travelling waves is close to $\sqrt{V^{\prime \prime}(0)}$. 


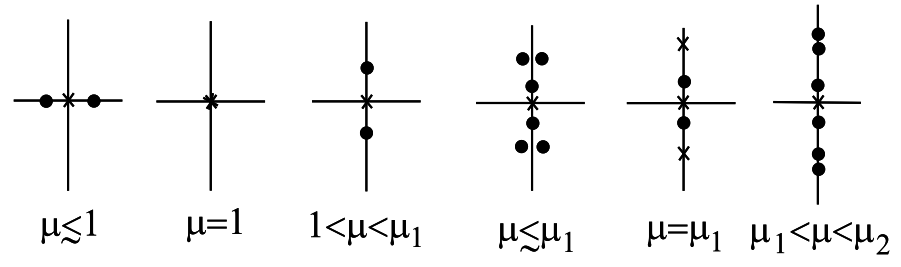

Figure 1: Position of eigenvalues of $L_{\mu}$ near the imaginary axis.

Using the center manifold reduction as in [4], we obtain in this simplest case a 4-dimensional reversible ODE. Next bifurcation, for $\mu$ near $\mu_{1}$, leads to a 8dimensional reversible ODE, and so on. The reduction argument follows exactly the same lines as in [4], so here we concentrate on the specific results on the reduced ODE, in the simplest case (4-dim).

We now need to construct the projection $P$ on the 4-dimensional subspace of $\mathbb{H}$, belonging to the quadruple eigenvalue $0\left(\mu=\mu_{0}=1\right)$, and which comutes with $L_{\mu_{0}}$. This projection is given by the Laurent expansion in $\mathcal{L}(\mathbb{H})$ of its resolvent operator near $\lambda=0$ (see [7])

$$
\left(\lambda \mathbb{I}-L_{\mu_{0}}\right)^{-1}=\frac{D^{3}}{\lambda^{4}}+\frac{D^{2}}{\lambda^{3}}+\frac{D}{\lambda^{2}}+\frac{P}{\lambda}-\widetilde{L}_{\mu_{0}}^{-1}-\lambda \widetilde{L}_{\mu_{0}}^{-2}-\ldots
$$

where $P$ is the projection we are looking for, and $D=L_{\mu_{0}} P$ is nilpotent $\left(D^{4}=\right.$ $0)$, and $\widetilde{L}_{\mu_{0}}^{-1}$ is the pseudo-inverse of $L_{\mu_{0}}$ on the subspace $(\mathbb{I}-P) \mathbb{H}$. The 4 dimensional subspace $P \mathbb{H}$ is spanned by the following vectors

$$
\begin{aligned}
\zeta_{0} & =(1,0,1)^{T}, \\
\zeta_{1} & =(0,1, v)^{T}, \\
\zeta_{2} & =\left(0,0, v^{2} / 2\right)^{T}, \\
\zeta_{3} & =\left(0,0, v^{3} / 6\right)^{T},
\end{aligned}
$$

which satisfy

$$
\begin{aligned}
& L_{\mu_{0}} \zeta_{0}=0, \quad S \zeta_{0}=-\zeta_{0}, \\
& L_{\mu_{0}} \zeta_{1}=\zeta_{0}, \quad S \zeta_{1}=\zeta_{1}, \\
& L_{\mu_{0}} \zeta_{2}=\zeta_{1}, \quad S \zeta_{2}=-\zeta_{2}, \\
& L_{\mu_{0}} \zeta_{3}=\zeta_{2}, \quad S \zeta_{3}=\zeta_{3} .
\end{aligned}
$$

After elementary computations, we obtain the following expression for the pro- 
jection $P$ :

$$
\begin{aligned}
P W & =\left((P W)_{x},(P W)_{\xi},(P W)_{X}\right)^{T} \\
& =(P W)_{x} \zeta_{0}+(D W)_{x} \zeta_{1}+\left(D^{2} W\right)_{x} \zeta_{2}+\left(D^{3} W\right)_{x} \zeta_{3}, \\
(P W)_{x} & =\frac{2}{5}\left\{f_{0}-\int_{0}^{1}\left[(1-s)-5(1-s)^{3}\right]\left[F_{2}(s)+F_{2}(-s)\right] d s\right\} \\
(D W)_{x} & =(P W)_{\xi}=\frac{2}{5}\left\{f_{1}-\int_{0}^{1}\left[1-15(1-s)^{2}\right]\left[F_{2}(s)-F_{2}(-s)\right] d s\right\}, \\
\left(D^{2} W\right)_{x} & =(D W)_{\xi}=-12\left\{f_{0}-\int_{0}^{1}(1-s)\left[F_{2}(s)+F_{2}(-s)\right] d s\right\} \\
\left(D^{3} W\right)_{x} & =\left(D^{2} W\right)_{\xi}=-12\left\{f_{1}-\int_{0}^{1}\left[F_{2}(s)-F_{2}(-s)\right] d s\right\} .
\end{aligned}
$$

where $W=\left(f_{0}, f_{1}, F_{2}\right)^{T} \in \mathbb{H}$. The expression for $D$ follows now immediately from its definition. In what follows, for sake of clarity, we denote by $\zeta_{j}^{*}$ the linear continuous forms on $\mathbb{H}$ given for any $W \in \mathbb{H}$ by

$$
\begin{aligned}
& \zeta_{0}^{*}(W)=(P W)_{x}, \\
& \zeta_{1}^{*}(W)=(D W)_{x}=\zeta_{0}^{*}\left(L_{\mu_{0}} W\right), \\
& \zeta_{2}^{*}(W)=\left(D^{2} W\right)_{x}, \\
& \zeta_{3}^{*}(W)=\left(D^{3} W\right)_{x}
\end{aligned}
$$

and we check easily that

$$
\begin{gathered}
\zeta_{j}^{*}(S W)=(-1)^{j+1} \zeta_{j}^{*}(W), \\
\zeta_{k}^{*}\left(\zeta_{j}\right)=\delta_{k j}, \quad k, j=0,1,2,3,
\end{gathered}
$$

where $\delta_{k j}=1$ if $k=j$, and $=0$ otherwise.

\section{Reduced system}

The system (3) is invariant under the following shift operator

$$
\tau_{q}: U \longmapsto \tau_{q} U=U+q \zeta_{0}, \quad \forall q \in \mathbb{R},
$$

which corresponds to the invariance of (2) under $x \longmapsto x+q$. Indeed, we check easily that

$$
L_{\mu} \tau_{q}=L_{\mu}, \quad M_{\tau} \circ \tau_{q}=M_{\tau} .
$$

It is then natural to decompose any $U \in \mathbb{H}$ as follows

$$
U=W+q \zeta_{0}, \quad \zeta_{0}^{*}(W)=0,
$$


and we denote by $\mathbb{H}_{1}$ the codimension one subspace of $\mathbb{H}$ where $W$ lies. We use the similar definition for the subspace $\mathbb{D}_{1}$ of $\mathbb{D}$. Noticing that $\zeta_{0}^{*}\left(0, f_{1}, 0\right)^{T}=0$, the system (3) becomes

$$
\begin{aligned}
\frac{d q}{d t} & =\zeta_{0}^{*}\left(L_{\mu_{0}} W\right)+0=\zeta_{1}^{*}(W), \\
\frac{d W}{d t} & =\widehat{L}_{\mu} W+M_{\tau}(W),
\end{aligned}
$$

where $\widehat{L}_{\mu} W=L_{\mu} W-\zeta_{1}^{*}(W) \zeta_{0}$. The operator $\widehat{L}_{\mu_{0}}$ as an operator acting in $\mathbb{H}_{1}$ has the same spectrum as $L_{\mu_{0}}$ except that 0 is now triple instead of quadruple. Indeed we check that

$$
\widehat{L}_{\mu_{0}} \zeta_{1}=0, \quad \widehat{L}_{\mu_{0}} \zeta_{2}=\zeta_{1}, \quad \widehat{L}_{\mu_{0}} \zeta_{3}=\zeta_{2}, \quad \zeta_{3}^{*}\left(\widehat{L}_{\mu_{0}} W\right)=0
$$

holds. We now use the center manifold reduction on the system (14) in $\mathbb{D}_{1}$. Applying a proof identical to the one given in [4], we know that the "small" solutions are contained in a center manifold which is as regular as $V^{\prime}$ in the original system (2), of the form

$$
W=A \zeta_{1}+B \zeta_{2}+C \zeta_{3}+\Phi_{\mu}(A, B, C),
$$

where $\mu$ is near $\mu_{0}$ and $(A, B, C)$ near 0 , and $\Phi$ is regular and at least quadratic in its set of arguments, taking values in $\mathbb{D}_{1}$, and such that $\Phi(0,0,0, \mu)=0$. Now, we observe that the linearized operator for $\mu=\mu_{0}$, is a $3 \times 3$ Jordan block, with 0 on the diagonal, and we notice that the reversibility symmetry $S$ reduced to the 3-dimensional invariant subspace has the following representation

$$
S_{0}:(A, B, C) \longmapsto(A,-B, C) .
$$

It then results from normal form theory [see for instance [3] p.25 and p.31 (exercice I.18 for the reversible vector field)] that we can choose coordinates $A, B, C$ in choosing a suitable form for $\Phi_{\mu}$ up to a certain order, such that for any fixed $p$ smaller than the degree of regularity of $V^{\prime}$, the system reads

$$
\begin{aligned}
& \frac{d A}{d t}=B, \\
& \frac{d B}{d t}=C+A \phi_{\mu}\left(A, B^{2}-2 A C\right)+R_{B}\left(A, B^{2}, C, \mu\right), \\
& \frac{d C}{d t}=B \phi_{\mu}\left(A, B^{2}-2 A C\right)+B R_{C}\left(A, B^{2}, C, \mu\right),
\end{aligned}
$$

where $\phi_{\mu}$ is a polynomial in its arguments, of degree $p$ in $(A, B, C)$, and

$$
\left|R_{B}\right|+\left|B R_{C}\right|=O\left\{(|A|+|B|+|C|)^{p+1}\right\} .
$$

For the sake of completeness we add the equation for $q$

$$
\frac{d q}{d t}=A+\zeta_{1}^{*}\left[\Phi_{\mu}(A, B, C)\right]
$$


In the appendix we compute the principal part of polynomial $\phi_{\mu}$

$$
\begin{aligned}
\phi_{\mu}\left(A, B^{2}-2 A C\right) & =\nu+a A+b\left(B^{2}-2 A C\right)+c A^{2}+\ldots \\
\nu & =6(1-\mu)+O\left[(1-\mu)^{2}\right], \\
a & =-8 \tau^{2} \beta[1+O(\nu)],
\end{aligned}
$$

and, if $\beta=0$, and $\gamma \neq 0$

$$
a=0, \quad b=(3 / 10) \tau^{2} \gamma[1+O(\nu)], \quad c=-9 \tau^{2} \gamma[1+O(\nu)] .
$$

In $\phi_{\mu}$ we consider all coefficients $(a, b, c, .$.$) as functions of \nu$ instead of $\mu$, for a better comfort. A nice property of $(16,17,18)$ is that if we suppress the higher order terms $R_{B}, R_{C}$ which are not in normal form, then this "truncated" system is integrable. Indeed, we have the two first integrals

$$
\begin{aligned}
B^{2}-2 A C & =K, \\
C-\Psi(A, K, \nu) & =H,
\end{aligned}
$$

where

$$
\Psi(A, K, \nu)=\int_{0}^{A} \phi_{\mu}(s, K) d s .
$$

For $(H, K)$ fixed, all trajectories in the $(A, B, C)$ space are given by

$$
\begin{aligned}
& B^{2}=f_{H, K}(A), \\
& \quad f_{H, K}(A) \stackrel{\text { def }}{=} K+2 H A+2 A \Psi(A, K, \nu), \\
& C=H+\Psi(A, K, \nu) .
\end{aligned}
$$

Let us start with the case when $a \neq 0$, which corresponds for the potential $V$ to assuming that $\beta \neq 0$. The corresponding curves $B^{2}=f_{H, K}(A)$ are deduced from figure 2 , depending on the values of first integrals $(H, K)$. In all cases we have a family of equilibria implicitly given by

$$
B=0, \quad C+A \phi_{\mu}(A,-2 A C)=0,\left(\text { i.e. } \partial_{A} f_{H, K}(A)=0\right),
$$

which correspond to the curve in the $(H, K)$ plane at figure 2 . These equilibria may be elliptic or hyperbolic depending on the branch $\Gamma_{e}$ or $\Gamma_{h}$ where $(H, K)$ is sitting. On the right branch, for $\nu>0$ and $H=K=0$, there is one solution homoclinic to 0 , and, on the same branch of the $(H, K)$ plane, the equilibria (then non zero) are also limit points of homoclinics. Other small bounded solutions are periodic, corresponding to the positive part of $f_{H, K}$ when the curve intersects transversally the axis $B=0$.

In the case $a=0$, we need to consider the curves of the $(A, B)$ plane given by

$$
B^{2}=\frac{2}{3} c A^{4}+2 \widetilde{\nu} A^{2}+2 H A+K,
$$

which is the principal part of $f_{H, K}(A)$, and where $\widetilde{\nu}=\nu+b K$. We give at figure 3 a sketch of the curve of equilibria $\Gamma$ in all cases when $\nu c \neq 0$ (denoting 


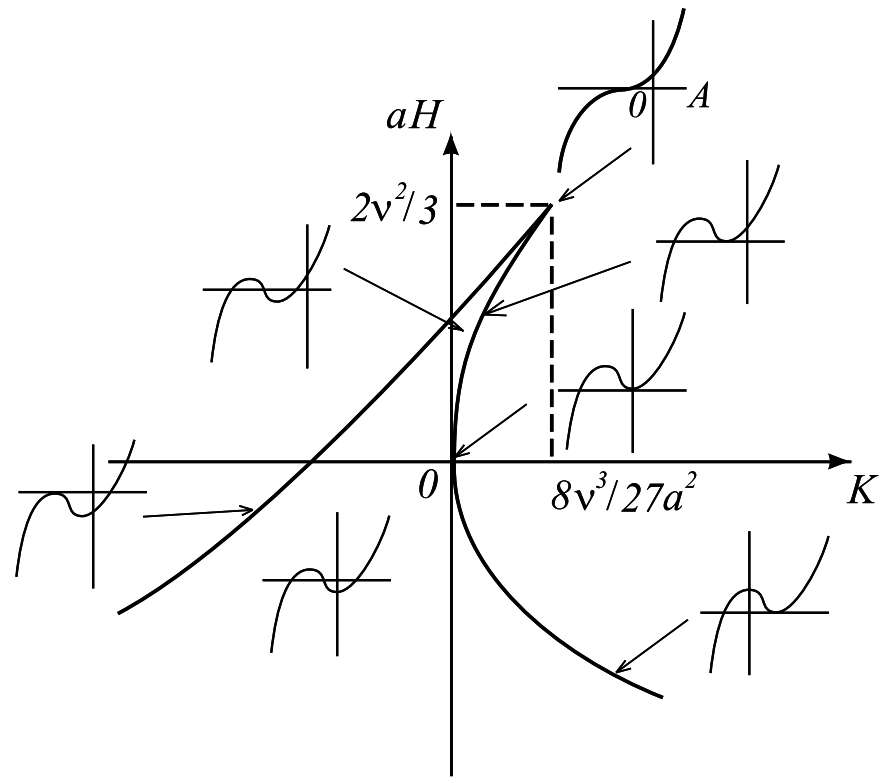

Figure 2: Different graphs of $A \longmapsto f_{H, K}(a A)$ for $\nu>0, a \neq 0$.

by $\Gamma_{e}$ and $\Gamma_{h}$ as above the elliptic or hyperbolic equilibria). We also draw the various graphs in the plane $\left(B^{2}, A\right)$ necessary for the study of small bounded solutions of $(16,17,18)$, truncated to its normal form. Notice that the sizes of $H$ and $K$ are respectively $O\left(|\nu|^{3 / 2}\right)$, and $O\left(\nu^{2}\right)$, which shows that $\widetilde{\nu} \sim \nu$. At figure 4 we give the phase portraits for non trivial cases (ii) $\nu>0, c<0$, and (iv) $\nu<0, c>0$, where we concentrate on bounded solutions for $t \in \mathbb{R}$, and we notice that a change $H \rightarrow-H$ is equivalent in these graphs, to the changing $A \rightarrow-A$. We notice that there are various homoclinics, two of them having a decay at infinity in $1 / t^{2}$ instead of exponential $\left(\nu>0, c<0, H= \pm H_{m}, K=K_{m}\right)$. We notice one heteroclinic cycle connecting two equilibria (both invariants under the reversibility symmetry) $\left(\nu<0, c>0, H=0, K=K_{0}\right)$.

Before giving a proof of the persistence of the various solutions we found on system $(16,17,18)$ truncated to its quadratic or cubic normal form, let us describe the form of the corresponding waves solutions of the original system (2), (1).

An equilibrium with $A_{e q} \neq 0$, gives $x(t) \sim q(t)=\widetilde{A}_{e q} t+q_{0}$, where $\widetilde{A}_{e q}$ is close to $A_{e q}$. This corresponds to solutions of (1) of the form $X_{n}(\widetilde{t})=\left(\widetilde{A}_{e q} / \tau\right)(n \tau-$ $\widetilde{t})+q_{0}$. Observing that a consequence is $X_{n+1}(\widetilde{t})-X_{n}(\widetilde{t})=\widetilde{A}_{e q}$ is constant, these solutions represent a uniform stretching (if $A_{e q}>0$ ) or contraction (if $\left.A_{e q}<0\right)$ of the lattice of particles.

A periodic solution in $(A, B, C)$ gives $x(t) \sim q(t)=\omega t+\Omega(t)$, with $\Omega$ periodic, i.e. a superposition of the uniform stretching (or compression) with 
a periodic oscillation. Notice that there are cases (choosing suitably $(H, K)$ ) where $\omega$ cancels ( $\omega$ is close to the average of $A$ ). In these last cases, this leads to time periodic solutions of (2).

A solution homoclinic to 0 , corresponds to a localized solution of (2), since $\dot{q}$ also tends to 0 exponentially in time. We obtain such homoclinics for $\beta \neq$ $0, \nu>0$, and for $\beta=0, \gamma>0, \nu>0$. this last result is in accordance with the results of [2], only interested into localized solutions.

We also obtain solutions homoclinic to non zero equilibria (for $\beta \neq 0, \forall \nu \neq 0$, and for $\beta=0, \gamma \nu>0$ ), corresponding to the family of uniform stretching or compression between particles at infinity. The particular cases $H= \pm H_{m}, K=$ $K_{m}$ give a convergence in $1 / t^{2}$ at infinity for $A$. Then the convergence to a constant of $x(t)-\widetilde{A}_{\text {eq }} t$ as $t \rightarrow \pm \infty$ is in $1 / t$.

Finally, the heteroclinic solution connects two elements of this basic family of solutions of stretched type at one infinity, and of compression type at the other infinity!

\section{Persistence of Homoclinics}

In this section, we give details of the proof of existence of homoclinics close to the homoclinic solutions of the normal form.

\subsection{Case $a \neq 0, \nu>0$}

For the normal form truncated at quadratic order, let us make the rescaling

$$
A=\frac{\nu}{a} \underline{A}, B=\frac{\sqrt{2} \nu^{3 / 2}}{a} \underline{B}, C=\frac{2 \nu^{2}}{a} \underline{C}, t=\frac{\tau}{\sqrt{2 \nu}}
$$

which gives the homoclinic solution under the form

$$
\begin{aligned}
H(\tau) & =[\underline{A}(\tau), \underline{B}(\tau), \underline{C}(\tau)]^{T}=\left[h(\tau), \dot{h}(\tau), h_{C}(\tau)\right]^{T}, \\
h(\tau) & =-2 / \cosh ^{2} \tau / 2, h_{C}=h / 2+h^{2} / 4 .
\end{aligned}
$$

This basic homoclinic $H$ satisfies $S H(-\tau)=H(\tau)$ (it is a "reversible" solution). We are now looking for a reversible solution of the full system $(16,17,18)$ close to $H$.

Let us pose $[\underline{A}(\tau), \underline{B}(\tau), \underline{C}(\tau)]^{T}=H+Y$, then $Y=(u, v, w)^{T}$ satisfies

$$
\frac{d Y}{d \tau}-\mathcal{L}(\tau) Y=M(\nu, \tau, Y)
$$

with

$$
\mathcal{L}(\tau)=\left(\begin{array}{ccc}
0 & 1 & 0 \\
\frac{1}{2}+h & 0 & 1 \\
\grave{h} / 2 & \frac{1}{2}(1+h) & 0
\end{array}\right), \quad S=\left(\begin{array}{ccc}
1 & 0 & 0 \\
0 & -1 & 0 \\
0 & 0 & 1
\end{array}\right)
$$


and

$$
\begin{aligned}
S M(\nu, \tau, Y) & =-M(\nu,-\tau, S Y), \\
M(\nu, \tau, 0) & =0, \quad D_{Y} M(\nu, \tau, 0)=0, \\
\|M(\nu, \tau, Y)\| & =O\left(\nu(\|H\|+\|Y\|)^{3}+\|Y\|^{2}\right) .
\end{aligned}
$$

Let us define Banach spaces $C_{-\lambda, S}^{0}$ and $C_{-\lambda, A}^{0}$, for any $\lambda \in \mathbb{R}$ :

$$
\begin{aligned}
& C_{-\lambda, S}^{0}=\left\{Z \in C^{0}\left(\mathbb{R}, \mathbb{R}^{3}\right) ; S Z(-\tau)=Z(\tau), \sup _{\tau \in \mathbb{R}}|| Z(\tau) \| e^{\lambda|\tau|}<\infty\right\}, \\
& C_{-\lambda, A}^{0}=\left\{Z \in C^{0}\left(\mathbb{R}, \mathbb{R}^{3}\right) ; S Z(-\tau)=-Z(\tau), \sup _{\tau \in \mathbb{R}}|| Z(\tau)|| e^{\lambda|\tau|}<\infty\right\},
\end{aligned}
$$

equipped with the norm $\|Z\|_{\lambda}=\sup _{\tau \in \mathbb{R}}|| Z(\tau)|| e^{\lambda|\tau|}$.

We look for $Y$ solution of (24) in $C_{-1, S}^{0}$. We notice that $Y \longmapsto M(\nu, \cdot, Y)$ is as regular as the given function $V^{\prime}$ (analytic for example) in (2) from $C_{-1, S}^{0}$ into $C_{-2, S}^{0}$ (see the estimate on $M$ ). The existence proof of the homoclinic solution follows directly from the implicit function theorem, once we prove that the linear operator $\frac{d}{d t}-\mathcal{L}(\tau)$ has a bounded inverse from $C_{-\lambda, A}^{0}$ to $C_{-1, A}^{0}$ for any $\lambda>1$.

For inverting this linear operator, we need three independent solutions of the linear homogeneous system

$$
\frac{d Y}{d t}-\mathcal{L}(\tau) Y=0
$$

with no restriction on symmetry and behavior at infinity. This system is integrable, as the original normal form, and we already know a solution which is $\dot{H}(\tau)$, due to time shift invariance. We notice that

$$
S \dot{H}(-\tau)=-\dot{H}(\tau), \quad \dot{H} \in C_{-1, A}^{0} .
$$

It is easy to find two other solutions of $(25)$ :

$$
\begin{aligned}
& P=\left[u_{1}, \dot{u}_{1}, \frac{u_{1}}{2}(1+h)\right]^{T}, \\
& Q=\left[u_{2}, \dot{u}_{2}, \frac{u_{2}}{2}(1+h)+1\right]^{T},
\end{aligned}
$$

with

$$
\begin{aligned}
& u_{1}(\tau)=\dot{h}(\tau) \int_{0}^{\tau}\left[s^{-2}-\dot{h}^{-2}(s)\right] d s+\dot{h}(\tau) / \tau, \\
& u_{2}(\tau)=\dot{h}(\tau) \int_{0}^{\tau} u_{1}(s) d s-h(\tau) u_{1}(\tau) .
\end{aligned}
$$


We notice that $u_{1}$ and $u_{2}$ are regular and even, $u_{1}(0)=1, u_{2}(0)=2, u_{1}(\tau) \sim$ $e^{|\tau|}, \quad u_{2}(\tau) \rightarrow-1$, as $\tau \rightarrow \pm \infty$. It results that

$$
\begin{array}{ll}
S P(-\tau)=P(\tau), & P \in C_{1, S}^{0}, \\
S Q(-\tau)=Q(\tau), & Q \in C_{0, S}^{0} .
\end{array}
$$

Let us show the following

Lemma 2 Let $\lambda>1$, then the linear operator $\frac{d}{d t}-\mathcal{L}(\tau)$ has a bounded inverse from $C_{-\lambda, A}^{0}$ into $C_{-1, S}^{0}$.

Proof. We first notice that for any $\tau,\{\dot{H}, P, Q\}$ forms a basis of $\mathbb{R}^{3}$, this is a consequence of the wronskian identity which gives here

$$
(\dot{H}, P, Q)=\operatorname{det}(\dot{H}, P, Q)=-1 .
$$

Let us decompose $Y$ as follows, which is always possible for functions having values in $\mathbb{R}^{3}$ :

$$
Y=r \dot{H}+p P+q Q,
$$

then the unique solution $Y \in C_{-1, S}^{0}$ of

is given by

$$
\frac{d Y}{d t}-\mathcal{L}(\tau) Y=G \in C_{-\lambda, A}^{0}
$$

$$
\begin{aligned}
& p(\tau)=\int_{\tau}^{\infty}(G(s), Q(s), \dot{H}(s)) d s, \quad \text { (even) } \\
& q(\tau)=\int_{\tau}^{\infty}(G(s), \dot{H}(s), P(s)) d s, \quad \text { (even) } \\
& r(\tau)=-\int_{0}^{\tau}(G(s), P(s), Q(s)) d s \quad \text { (odd). }
\end{aligned}
$$

It is clear that there is a positice number $c_{\lambda}$ such that

$$
\|Y\|_{1} \leq c_{\lambda}\|G\|_{\lambda}, \quad \forall \lambda>1
$$

holds. Notice that $\lambda=1$ is not good because of the estimate on $r(\tau)$ which would lead to a growing in $|\tau|$ at infinity. Observe that we only need $\lambda=2$ here, because of a complete account in $H$ of the linear terms in system $(16,17,18)$. This ends the proof of the lemma.

\subsection{Case $a=0, \nu>0, c<0$}

The two homoclinics to 0 , obtained for $\nu>0$ on the cubic normal form, are as follows

$$
\begin{aligned}
H(t) & =(A, B, C)^{T}=\left(h, \dot{h}, h_{C}\right)^{T}, \\
h(t) & =\sqrt{-3 \nu / c}(\cosh \sqrt{2 \nu} t)^{-1}, \\
h_{C} & =\nu h+c h^{3} / 3 .
\end{aligned}
$$


The cubic normal form linearized at $H(t)$ is again integrable (this comes immediately from the integrability of the normal form). We then proceed with the same method as at previous section. The properties of the basis of solutions of the homogeneous linear system are identical, concerning their symmetries and exponential behavior at infinity (modulo adapting the exponent). We notice that the term with the factor $\left(B^{2}-2 A C\right)$ may be pushed into the rest, since its size is smaller than the principal part (once scaling are made, the rest $M$ is here $\left.O\left(\sqrt{|\nu|}(\|H\|+\|Y\|)^{3}+\|Y\|^{2}\right)\right)$. The proof of persistence is then the same as above. We then established the following

Theorem 3 For $\varepsilon>0$ small enough, we consider the system (2) for $1-\varepsilon<$ $\tau^{2} V^{\prime \prime}(0)<1$, i.e. for velocities of the waves slightly above the first critical value ("sound velocity").

(i) In the case when $V^{\prime \prime \prime}(0) \neq 0$, there exist one and only one "small" localized solution of size $O\left[1-\tau^{2} V^{\prime \prime}(0)\right]$ with an exponential decay at infinity. If $V^{\prime \prime \prime}(0)<0$ the localized wave is of "local compression" type, while if $V^{\prime \prime \prime}(0)>0$ it is of "local stretching" type. Moreover, this solution is odd, and we can give its Taylor expansion in powers of the bifurcation parameter $1-\tau^{2} V^{\prime \prime}(0)$.

(ii) In the case when $V^{\prime \prime \prime}(0)=0, V^{(4)}(0)>0$, there exist two and only two "small" localized solutions of size $O\left(\left[1-\tau^{2} V^{\prime \prime}(0)\right]^{1 / 2}\right)$. They are both monotonous and odd. One wave is of "compression" type, while the other is of "stretched" type. Their decay at infinity is exponential, and we can give the Taylor expansion of these solutions in powers of the bifurcation parameter $\left[1-\tau^{2} V^{\prime \prime}(0)\right]$. In the case when $V^{(4)}(0)<0$ there is no small localized solution.

The oddness of these localized solutions comes from the reversibility of the solutions projected on the $x$ component. We have $x(t) \sim \int_{0}^{t} A(s) d s$, hence since the sign of $A$ is constant for each of the homoclinic solutions (see figures 2 and $4), x$ is a monotonic function and the results about compression or stretching follows directly from an examination of the sign of $A$. At $\pm \infty, x(t)$ tends towards opposite limits.

Remark 4 Our theorem above should be compared with theorem 1 of G.Friesecke, J.A.Wattis [2]. These authors used a variational approach for obtaining nice global results on existence of localized solutions which have the properties of our local solutions. Our method is only local, but its advantage is that it is constructive, and valid for generic potentials $V$. In addition, as we see below, we obtain ALL "small" waves, solutions of (2), i.e. not only the localized ones.

\subsection{Other homoclinic solutions}

We observed on the normal form, at section 3 that there are hyperbolic equilibria other than the origin, which are the end points of homoclinic orbits. We show below how to prove the persistence of corresponding solutions for the system (2), which are localized if we forget the uniform compression or expansion between particles at infinity. We include here the study of the special cases where the 
equilibrium is a saddle-node and the homoclinic has just a polynomial decay at infinity ( $\operatorname{cases} \nu>0, c<0, K=K_{m}, H= \pm H_{m}$ ).

In the case of hyperbolic equilibria (invariants under $S$ ), the method is exactly the same as the one we follow when this point is the origin (previous section). The only technicallity comes from the necessity to take into account the full vector field $(16,17,18)$ for defining the new origin and the full linear terms around this new origin.

Let us consider for example the case $a \neq 0$. Equilibria of $(16,17,18)$ are of the form $\left(A_{0}, 0, C_{0}\right)$ where $C_{0}$ is determined via the implicit function theorem from equation

$$
C_{0}+A \phi_{\mu}\left(A_{0},-2 A_{0} C_{0}\right)+R_{B}\left(A_{0}, 0, C_{0}, \mu\right)=0
$$

which leads to

$$
C_{0}=-\nu A_{0}-a A_{0}^{2}+O\left(A_{0}^{3}\right) .
$$

The linearized operator at these equilibria is now given by

$$
\left(\begin{array}{ccc}
0 & 1 & 0 \\
\nu+2 a A_{0}+O\left(A_{0}^{2}\right) & 0 & 1+O\left(A_{0}^{2}\right) \\
0 & \nu+a A_{0}+O\left(A_{0}^{2}\right) & 0
\end{array}\right)
$$

which anticommutes with $S$ and which has a zero eigenvalue. Moreover it is a regular perturbation of the $3 \times 3$ Jordan block matrix. Hence, we use again normal form technique to transform the linear part as well as the nonlinear part of the vector field into a quadratic normal form close to the quadratic normal form we had originally in $(16,17,18)$. Since $A_{0}$ is at most of order $\nu$ coefficients of the new normal form are at order $\nu$ close to the old ones. This may be a big change for the status of eigenvalues near 0 , but we already know the status of such eigenvalues from the previous study (see figure 2). Redefining a new parameter $\nu^{\prime}$ to mimic the proof of previous section, we can then proceed in exactly the same way for proving the persistence of all the homoclinic orbits we found on the normal form, provided they end to a hyperbolic equilibrium, and provided that we are not close to the singular case of the heteroclinic orbit for the cubic normal form of the case $a=0, c>0, \nu<0, H=0, K=K_{0}$.

Now arises the special case when the equilibrium is not hyperbolic. We found that, for $a=0, \nu>0, c<0$, this situation happens when $A_{0}$ is a triple root of $f_{H_{m}, K_{m}}(A)=0$. This corresponds to a linearized operator at this equilibrium with a triple 0 eigenvalue and a full $3 \times 3$ Jordan block. Let us use the same trick as above. First we show that such type of equilibria still exist for the full vector field (implicit function theorem for solving in $(C, \nu)$ as a function of $A_{0}$, then invert $A_{0}$ in function of $\nu$, in choosing its negative root $A_{0}=-\sqrt{\nu /(-2 c)}+O(\nu)$ (which corresponds to the case $H=H_{m}$ ). The linearized operator at this equilibrium has a triple 0 eigenvalue by construction. Using the same technicalities as above, we can produce a new cubic normal form which is a perturbation of the one in $(16,17,18)$, but with an unperturbed linear part (corresponding to $\nu=0$ in this previous system). This new normal form introduces a quadratic 
coefficient of order $\sqrt{\nu}$. This forces to consider also cubic coefficients which lead to terms of the same order, and which are small perturbations of the cubic terms of $(16,17,18)$. Now the truncated system is integrable, as before, and the homoclinic solution is still even for its $A$ component denoted by $\widetilde{A}$ with $\widetilde{A}(t)=A(t)-A_{0} \sim \frac{3}{\sqrt{-2 c \nu}} t^{-2}$ as $t \rightarrow \pm \infty$. Now we use the method we developed in [5] on another example of reversible system with a non hyperbolic limit point of homoclinic solution. The proof follows roughly the same lines as above, except that we need adapted spaces of continuous functions with the suitable decay at infinity, (here polynomial), specially in a lemma analogous to our lemma 2 above.

\subsection{Heteroclinic cycle}

In the case $a=0, c>0, \nu<0$, we find for the cubic normal form in $(16,17,18)$ the heteroclinic cycle

$$
\begin{aligned}
B^{2} & =2 \nu A^{2}+\frac{2 c}{3} A^{4}+K_{0}, \\
C & =\nu A+\frac{c}{3} A^{3},
\end{aligned}
$$

with $K_{0}=\frac{3 \nu^{2}}{2 c}$ and ending at points $\left( \pm A_{0}, 0, \pm C_{0}\right), A_{0}=\sqrt{-3 \nu / 2 c}, C_{0}=\frac{\nu}{2} A_{0}$. Notice that one branch is the image of the other by the symmetry $S$. In the above expressions, we did not consider in $(16,17,18)$ the terms with the factor $\left(B^{2}-2 A C\right)=K$, because this factor is of order $O\left(\nu^{2}\right)$, and the corresponding terms in the normal form are of higher order than the main ones (so we push these terms in the rests $R_{B}$ and $R_{C}$ ). The above end points belong to the one parameter family of equilibria for system $(16,17,18)$, given, via implicit function theorem, by

$$
\begin{aligned}
A & =A_{e}, B=0, C_{e}=C\left(A_{e}\right), \\
0 & =C_{e}+\nu A_{e}+c A_{e}^{3}+R_{B}\left(A_{e}, 0, C_{e}\right) .
\end{aligned}
$$

For proving the persistence of the heteroclinic cycle for the full vector field, the idea is to study the intersection points of each unstable manifold of the equilibria near $\left(-A_{0}, 0,-C_{0}\right)$ with the plane $A=0$. These intersections describe a curve $\Gamma_{-}$when $A_{e}$ varies near $-A_{0}$. In the same way we study the intersection points of each stable manifolds ending at equilibria near $\left(A_{0}, 0, C_{0}\right)$ with the plane $A=0$. They also describe a curve $\Gamma_{+}$when $A_{e}$ varies near $A_{0}$. We then prove that these two curves intersect transversally.

Let us consider the family of unstable manifolds of equilibria close to $\left(-A_{0}, 0,-C_{0}\right)$. For the cubic normal form, these curves, parameterized by $A$, are given by

$$
\begin{aligned}
B^{2} & =2 \nu A^{2}+\frac{2 c}{3} A^{4}+2 A H+K, \\
C & =\nu A+\frac{c}{3} A^{3}+H,
\end{aligned}
$$


where $H$, and $K$ are linked by the fact that for $A_{e}$

$$
\begin{aligned}
& H=-2 \nu A_{e}-\frac{4 c}{3} A_{e}^{3}, \\
& K=\frac{2 c}{3} A_{e}^{4}
\end{aligned}
$$

holds. In the plane $A=0$, the curve $\Gamma_{-}$is then given by $(B, C)=\left[\sqrt{K\left(A_{e}\right)}, H\left(A_{e}\right)\right]$ for $A_{e}$ close to $-A_{0}$. In the same way, the curve $\Gamma_{+}$is given by the same form, but for $A_{e}$ close to $A_{0}$. The two tangents to $\Gamma_{-}$and $\Gamma_{+}$at the intersection point $(B, C)=\left(\sqrt{K_{0}}, 0\right)$ are parallel to the following directions: $( \pm 2 \sqrt{-\nu}, 4 \nu)$. This shows the transversallity of the intersection of $\Gamma_{-} \cap \Gamma_{+}$for the cubic normal form. We need now to prove that the perturbation introduced by taking account of the full vector field $(16,17,18)$, does not break this transversallity. This is not obvious since the size of the perturbation is fixed, and not arbitrary small. However, after a suitable rescaling, letting the heteroclinic cycle of the normal form of order 1 , as well as the exponential decay at infinity, the perturbation terms of the rescaled system are $O(\sqrt{\nu})$, and the proof of persistence is straightforward with the transversallity argument. Let us sum up our results with the following

Theorem 5 For $\varepsilon>0$ small enough, we consider the system (2) for $1-\varepsilon<$ $\tau^{2} V^{\prime \prime}(0)<1+\varepsilon$ (velocities of the waves near the "sound velocity").

(i) For $V^{\prime \prime \prime}(0) \neq 0$, there is a one parameter family of "mainly localized" small solutions. Their size is $O\left(\left|1-\tau^{2} V^{\prime \prime}(0)\right|\right)$. The function $t \longmapsto x(t)$ is not always monotonous, and at $\pm \infty$ we have $x(t)-A_{e} t \rightarrow x_{0}^{ \pm}$, the convergence being exponential. For $A_{e}>0$, the limit at both infinities is a uniform stretching, while if $A_{e}<0$, the limit at both infinities is a uniform compression.

(ii) For $V^{\prime \prime \prime}(0)=0$, and $V^{(4)}(0)>0, \tau^{2} V^{\prime \prime}(0)<1$, or $V^{(4)}(0)<0, \tau^{2} V^{\prime \prime}(0)>$ 1 , there is a one parameter family of "mainly localized" solutions. Their size is $O\left(\left|1-\tau^{2} V^{\prime \prime}(0)\right|^{1 / 2}\right)$.

(iii) For $V^{\prime \prime \prime}(0)=0$, and $V^{(4)}(0)>0, \tau^{2} V^{\prime \prime}(0)<1$, there are two "mainly localized" solutions, of the type above, but whose convergence at infinity is in $1 /|t|$ (then not exponential). One of these solutions has a uniform stretching limit, the other has a uniform compression limit.

(iv) For $V^{\prime \prime \prime}(0)=0$, and $V^{(4)}(0)<0, \tau^{2} V^{\prime \prime}(0)>1$, there is a heteroclinic cycle of two solutions. One of them has a uniform compression at $-\infty$ and a uniform stretching at $+\infty$. It is the opposite for the other solution.

\section{Persistence of periodic solutions}

We consider the system $(16,17,18)$ in the case when the normal form truncated at quadratic order if $a \neq 0$, or at cubic order if $a=0, c \neq 0$, possesses periodic solutions. They constitute a two-parameter family because of the arbitrariness of the choice of $(H, K)$. Notice that $B$ cancels twice on each of these solutions, the branch for $B>0$ being mapped by $S$ into the branch $B<0$. The idea is 
then to only consider half of these solutions, i.e. their branch such that $B>0$. We then complete the solution by applying the symmetry $S$, like this, we build "reversible" periodic solutions.

We first eliminate time and consider the corresponding solutions parameterized by $A$, in the plane $\left(B^{2}, C\right)$, for $B>0$, passing through end points such that $B=0$. Let us consider, for instance the case $a \neq 0, \nu>0$, and rescale as in section 4.1. Setting $B^{2}=u$, we obtain the following system in the $(u, \underline{C})$ plane:

$$
\begin{aligned}
& \frac{d \underline{C}}{d \underline{A}}=\frac{1}{2}(1+\underline{A})+\nu \rho_{1}(\underline{A}, u, \underline{C}, \nu), \\
& \frac{d u}{d \underline{A}}=2 \underline{C}+\underline{A}+\underline{A}^{2}+\nu \rho_{2}(\underline{A}, u, \underline{C}, \nu),
\end{aligned}
$$

where $\rho_{1}$ and $\rho_{2}$ are regular functions in their arguments. Now consider one solution of the unperturbed system

$$
\begin{aligned}
u & =\underline{A}^{2}+\frac{1}{2} \underline{A}^{3}+2 \underline{H A}+\underline{K}, \\
\underline{C} & =\frac{1}{2} \underline{A}+\frac{1}{4} \underline{A}^{2}+\underline{H},
\end{aligned}
$$

for values of $(\underline{H}, \underline{K})$ such that this solution connects two points given by $\underline{A}=$ $\underline{A}_{1}, \underline{C}=\underline{C}_{1}$ and $\underline{A}=\underline{A}_{2}, \underline{C}=\underline{C}_{2}$ where $u=0$, and where $u>0$ in between (see the form of the cubic at figure 2 ). We also assume that these end points are not equilibria, since we deal with periodic solutions (not homoclinic). This means that $\frac{d u}{d \underline{A}}$ does not cancel at $\underline{A}=\underline{A}_{1}$ or $\underline{A}_{2}$. Now consider the full (perturbed) system, and start at point $\underline{A}=\underline{A}_{1}$ with $(u, \underline{C})=\left(0, \underline{C}_{1}\right)$. The classical perturbation result on ordinary differential equations, shows that there is a trajectory near the unperturbed one, and reaching after a finite interval of $\underline{A}^{\prime} s$ ( $\underline{A}$ near $\underline{A}_{2}$ ) the line $u=0$, near $\underline{C}=\underline{C}_{2}$. This proof is valid as soon as we are not too close to a homoclinic curve, i.e. the starting point has to be not too close to an equilibrium. Completing the trajectory in the $(\underline{A}, \underline{B}, \underline{C})$ space by symmetry $S$, then gives a reversible periodic solution. So we indeed have a two parameter family of such solutions in all cases, except for $a=0, \nu>0, c>0$ (see figure 3 case (i)). Let us sum up this result in the following

Theorem 6 For $\varepsilon>0$ small enough, we consider the system (2) for $1-\varepsilon<$ $\tau^{2} V^{\prime \prime}(0)<1+\varepsilon$ (velocities of the waves near the "sound velocity").

(i) For $V^{\prime \prime \prime}(0) \neq 0$, there is a two parameter family of periodic small solutions. Their size is $O\left(\left|1-\tau^{2} V^{\prime \prime}(0)\right|\right)$.

(ii) For $V^{\prime \prime \prime}(0)=0$, and $V^{(4)}(0)>0, \tau^{2} V^{\prime \prime}(0)<1$, or $\tau^{2} V^{\prime \prime}(0)>1$, there is a two parameter family of periodic solutions. Their size is $O\left(\left|1-\tau^{2} V^{\prime \prime}(0)\right|^{1 / 2}\right)$.

The average of each periodic solution is a uniform compression or a uniform stretching of the lattice or the basic uniform state $(x=0)$.

\section{Appendix}

In this appendix, we compute the coefficients of $\phi_{\mu}$ as mentioned in (20). 


\subsection{Linear coefficients}

The linear operator for the system $(16,17,18)$ takes the form

$$
\left(\begin{array}{lll}
0 & 1 & 0 \\
\nu & 0 & 1 \\
0 & \nu & 0
\end{array}\right)
$$

whose eigenvalues are 0 and $\pm \sqrt{2 \nu}$. A simple identification with the eigenvalues $\lambda$ close to 0 , given by the dispersion relation $N(\lambda ; \mu)=0$, for $\mu$ close to 1 , leads to

$$
\nu=6(1-\mu)+O\left[(1-\mu)^{2}\right]
$$

In fact, we can also compute the linear terms of order $\nu$ in $\Phi_{\mu}$ denoted by $\nu A \Phi_{100}^{(1)}+\nu B \Phi_{010}^{(1)}+\nu C \Phi_{001}^{(1)}$. They are solution of the following system, obtained after identification between linear terms in (14), after using $(15)$ and $(16,17,18)$

$$
\begin{aligned}
\zeta_{2}-\widehat{L}_{\mu_{0}} \Phi_{100}^{(1)} & =-\nu \Phi_{010}^{(1)}-\nu \widehat{\mu} L^{(1)} \Phi_{100}^{(1)}, \\
\zeta_{3}+\Phi_{100}^{(1)}-\widehat{L}_{\mu_{0}} \Phi_{010}^{(1)}+\widehat{\mu}(0,1,0)^{T} & =-\nu \Phi_{001}^{(1)}-\nu \widehat{\mu} L^{(1)} \Phi_{010}^{(1)}, \\
\Phi_{010}^{(1)}-\widehat{L}_{\mu_{0}} \Phi_{001}^{(1)} & =-\nu \widehat{\mu} L^{(1)} \Phi_{001}^{(1)},
\end{aligned}
$$

where $\widehat{\mu}=(1-\mu) / \nu$ and where we defined $\widehat{L}^{(1)}$ by $\widehat{L}_{\mu}=\widehat{L}_{\mu_{0}}-\nu \widehat{\mu}(\nu) \widehat{L}^{(1)}$. Notice that we already know that $\widehat{\mu}(0)=1 / 6$.

All coefficients are functions of $\nu$, and they are uniquely determined by the implicit function theorem, once we add the conditions $\zeta_{0}^{*}\left(\Phi_{i j k}\right)=\zeta_{1}^{*}\left(\Phi_{i j k}\right)=0$, $i+j+k=1$, and $S \Phi_{i j k}=(-1)^{j+1} \Phi_{i j k}$ We obtain for $\nu=0$

$$
\begin{aligned}
& \Phi_{100}^{(1)}=\zeta_{3}, \\
& \Phi_{010}^{(1)}=k \zeta_{0}+\left(0,0, v^{4} / 12\right)^{T}, \quad k=13 / 12600 \\
& \Phi_{001}^{(1)}=k \zeta_{1}+\left(0,0, v^{5} / 60\right)^{T} .
\end{aligned}
$$

\subsection{Quadratic coefficients}

Defining quadratic coefficients of $\Phi_{\mu}$ by $\sum_{i+j+k=2} \Phi_{i j k} A^{i} B^{j} C^{k}$, where, as above, $\Phi_{i j k}$ are functions of $\nu$, we identify quadratic terms in the same way as above

$$
\begin{aligned}
a \zeta_{2} & =\widehat{L}_{\mu_{0}} \Phi_{200}-\nu \widehat{\mu}(\nu) \widehat{L}^{(1)} \Phi_{200}-\nu \Phi_{110}-a \nu \Phi_{010}^{(1)} \\
a \zeta_{3}+2 \Phi_{200} & =\widehat{L}_{\mu_{0}} \Phi_{110}-\nu \widehat{\mu}(\nu) \widehat{L}^{(1)} \Phi_{110}-\nu \Phi_{101}-2 \nu \Phi_{020}-a \nu \Phi_{001}^{(1)}+ \\
& +\left(0,2 \tau^{2} \beta, 0\right)^{T}+2 M_{\tau}^{(2)}\left(\nu \zeta_{3}, \zeta_{2}+\nu \Phi_{010}^{(1)}\right)+2 \nu M_{\tau}^{(2)}\left(\zeta_{1}, \Phi_{010}^{(1)}\right)
\end{aligned}
$$




$$
\begin{aligned}
\Phi_{110} & =\widehat{L}_{\mu_{0}} \Phi_{020}-\nu \widehat{\mu}(\nu) \widehat{L}^{(1)} \Phi_{020}-\nu \Phi_{011}, \\
\Phi_{110} & =\widehat{L}_{\mu_{0}} \Phi_{101}-\nu \widehat{\mu}(\nu) \widehat{L}^{(1)} \Phi_{101}-\nu \Phi_{011}, \\
2 \Phi_{020}+\Phi_{101} & =\widehat{L}_{\mu_{0}} \Phi_{011}-\nu \widehat{\mu}(\nu) \widehat{L}^{(1)} \Phi_{011}-2 \nu \Phi_{002}+\left(0, \tau^{2} \beta / 3,0\right)^{T}+ \\
& +2 M_{\tau}^{(2)}\left(\nu \Phi_{010}^{(1)}, \zeta_{3}+\nu \Phi_{001}^{(1)}\right)+2 \nu M_{\tau}^{(2)}\left(\zeta_{2}, \Phi_{001}^{(1)}\right), \\
\Phi_{011} & =\widehat{L}_{\mu_{0}} \Phi_{002}-\nu \widehat{\mu}(\nu) \widehat{L}^{(1)} \Phi_{002},
\end{aligned}
$$

where $M_{\tau}^{(2)}(V, V)=\beta \tau^{2}\left(0,\left(\delta^{1} X-x\right)^{2}-\left(x-\delta^{-1} X\right)^{2}, 0\right)^{T}$. Solving the system for $\nu=0$, leads to a unique solution $\left\{\Phi_{i j k}, a, k_{1}\right\}$ satisfying

$$
\begin{aligned}
& \zeta_{0}^{*}\left(\Phi_{i j k}\right)=0, i+j+k=2, \text { and } S \Phi_{i j k}=(-1)^{j+1} \Phi_{i j k}, \\
& \zeta_{1}^{*}\left(\Phi_{i j k}\right)=0, i+j+k=2, i \neq 2, \zeta_{1}^{*}\left(\Phi_{200}-k_{1} \zeta_{1}\right)=0 .
\end{aligned}
$$

Indeed we obtain

$$
\begin{aligned}
& \Phi_{200}=a \zeta_{3}+k_{1} \zeta_{1}, \quad a(0)=-8 \tau^{2} \beta, \quad k_{1}(0)=-(4 / 15) \tau^{2} \beta, \\
& \Phi_{110}=2 k_{1} \zeta_{2}+x_{110} \zeta_{0}+\left(0,0, a v^{4} / 8\right)^{T}, x_{110}=13 a / 8400 \\
& \Phi_{020}=\Phi_{101}=x_{110} \zeta_{1}+2 k_{1} \zeta_{3}+\left(0,0, a v^{5} / 40\right)^{T} \\
& \Phi_{011}=3 x_{110} \zeta_{2}+x_{011} \zeta_{0}+\left(0,0, k_{1} v^{4} / 4+a v^{6} / 80\right)^{T} \\
& \Phi_{002}=3 x_{110} \zeta_{3}+x_{011} \zeta_{1}+\left(0,0, k_{1} v^{5} / 20+a v^{7} / 560\right)^{T} .
\end{aligned}
$$

It then results by the implicit function theorem, that quadratic coefficients $\left\{\Phi_{i j k}, a, k_{1}\right\}$ are uniquely solvable in function of $\nu$, and satisfy $(29,30)$. It also results that in the case when $\beta=0$ (no quadratic term in $V^{\prime}$ ), then all quadratic coefficients cancel, as well in the reduced system $(16,17,18)$, as in $\Phi_{\mu}(a=0$ and $\left.\Phi_{i j k}=0, i+j+k=2\right)$.

\subsection{Cubic coefficients}

In this section we assume that the quadratic coefficient $\beta$ in $V^{\prime}$ cancels. It is then necessary to compute the coefficients $b$ and $c$ in $\phi_{\mu}(20)$. The cubic term in (14) is then given by

$$
M_{\tau}^{(3)}(V, V, V)=\left(0, \tau^{2} \gamma\left[\left(\delta^{1} X-x\right)^{3}-\left(x-\delta^{-1} X\right)^{3}\right], 0\right)^{T} .
$$

Here below we only compute the principal part for $\nu=0$, of the cubic coefficients $b$ and $c$ of the normal form $(16,17,18)$. Identification of the coeffients of $A^{3}, A^{2} B$, $A^{2} C, A B^{2}, A B C, B^{3}$ leads to the system

$$
\begin{aligned}
c \zeta_{2} & =\widehat{L}_{\mu_{0}} \Phi_{300}, \\
c \zeta_{3}+3 \Phi_{300} & =\widehat{L}_{\mu_{0}} \Phi_{210}+\left(0,3 \tau^{2} \gamma, 0\right)^{T}, \\
-2 b \zeta_{2}+\Phi_{210} & =\widehat{L}_{\mu_{0}} \Phi_{201}, \\
b \zeta_{2}+2 \Phi_{210} & =\widehat{L}_{\mu_{0}} \Phi_{120}, \\
-2 b \zeta_{3}+2 \Phi_{201}+2 \Phi_{120} & =\widehat{L}_{\mu_{0}} \Phi_{111}+\left(0, \tau^{2} \gamma, 0\right)^{T}, \\
b \zeta_{3}+\Phi_{120} & =\widehat{L}_{\mu_{0}} \Phi_{030}+\left(0, \tau^{2} \gamma / 4,0\right)^{T} .
\end{aligned}
$$


The first equation leads to $\Phi_{300}=c \zeta_{3}+k_{1} \zeta_{1}$, and applying $\zeta_{3}^{*}$ to equation (32), we obtain

$$
c=-9 \tau^{2} \gamma .
$$

Now combining (33) with (34), and (35) with (36) we have

$$
\begin{aligned}
-5 b \zeta_{2} & =\widehat{L}_{\mu_{0}}\left[2 \Phi_{201}-\Phi_{120}\right], \\
-5 b \zeta_{3}+2 \Phi_{201}-\Phi_{120} & =\widehat{L}_{\mu_{0}}\left[\Phi_{111}-3 \Phi_{030}\right]+\left(0, \tau^{2} \gamma / 4,0\right)^{T} .
\end{aligned}
$$

Proceeding as above for $c$, we obtain immediately

$$
b=\frac{3}{10} \tau^{2} \gamma .
$$

\section{References}

[1] E.Fermi, J.Pasta, S.Ulam. Studies of nonlinear problems. Los Alamos Scientific Lab. report LA-1940, 1955.

[2] G.Friesecke, J.A.Wattis. Existence theorem for solitary waves on lattices. Com. Math. Phys. 161, 391-418, 1994.

[3] G.Iooss, M.Adelmeyer. Topics in bifurcation theory and Applications. Adv. Ser. Nonlinear Dynamics, 3, World Sci. 1992.

[4] G.Iooss, K.Kirchgässner. Travelling waves in a chain of coupled nonlinear oscillators. Preprint INLN 99.18.

[5] G.Iooss. Existence d'orbites homoclines à un équilibre elliptique, pour un système réversible. C.R.Acad. Sci. Paris, 324, I, 993-997, 1997.

[6] K.Kirchgässner. Wave solutions of reversible systems and Applications. J.Diff.Equ. 45, 113-127, 1982.

[7] T.Kato. Perturbation theory for linear operators. Springer Verlag, 1966. 

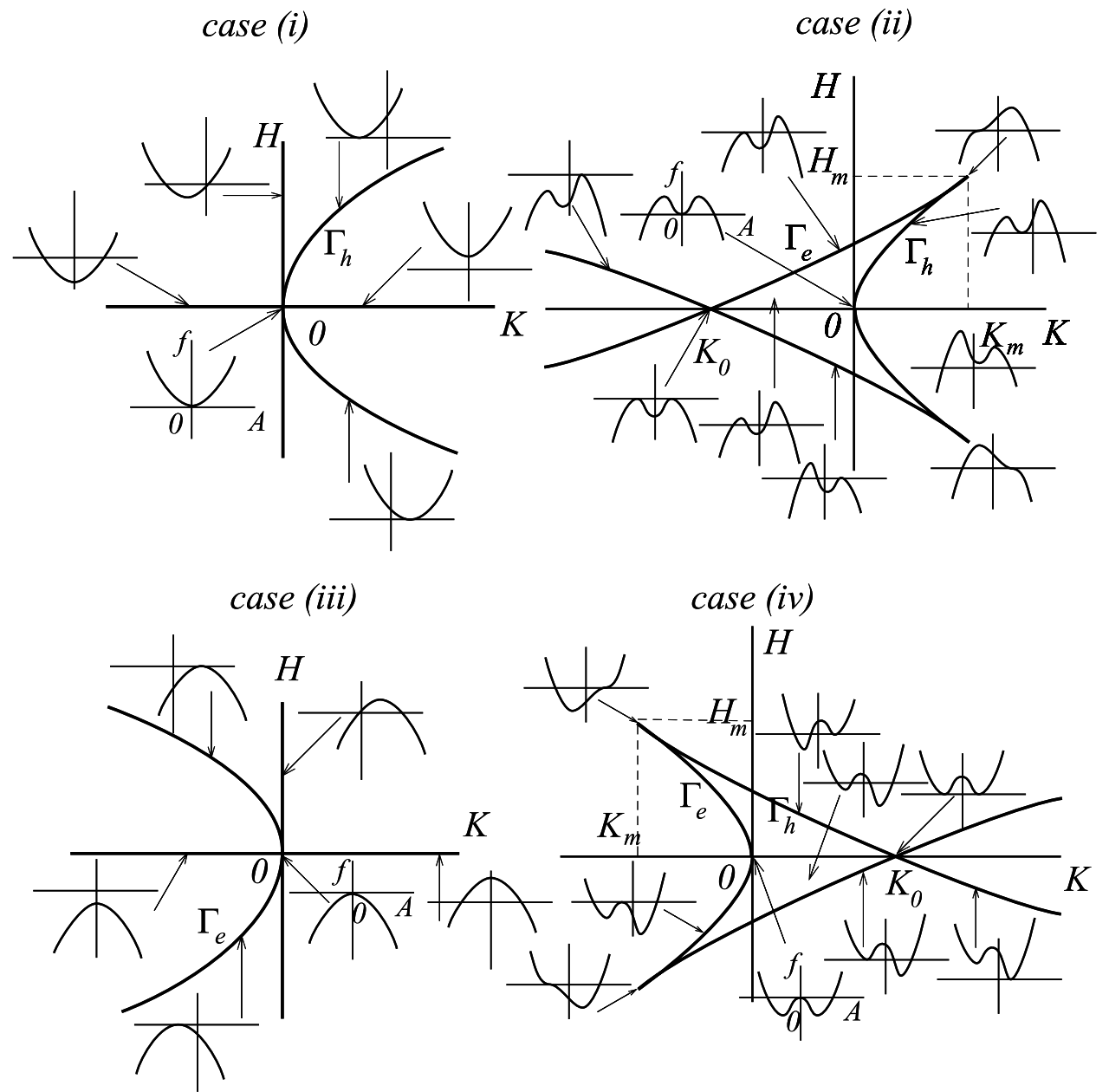

Figure 3: Curves $\Gamma$ of equilibria in $(H, K)$ planes, and graphs of $f_{H, K}(A)$. (i) $\nu>0, c>0$, (ii) $\nu>0, c<0$, (iii) $\nu<0, c<0$, (iv) $\nu<0, c>0$. $H_{m} \sim \frac{4}{3}|\nu| \sqrt{\frac{-\nu}{2 c}}, K_{m} \sim-\frac{\nu^{2}}{2 c}, K_{0} \sim 3 \nu^{2} / 2 c$. 


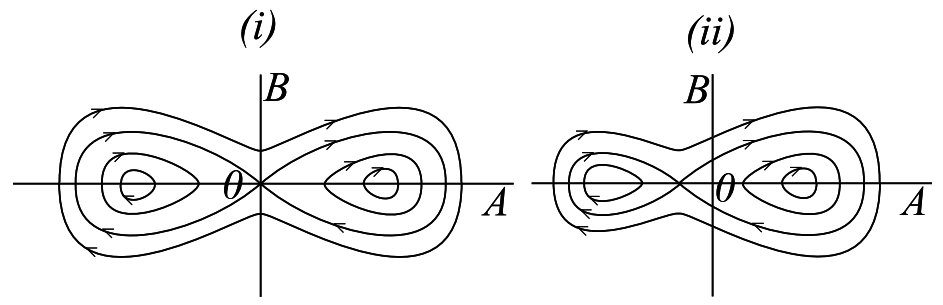

(iii)

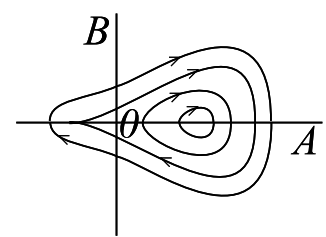

(v)

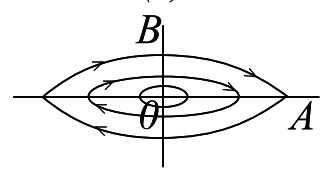

(iv)
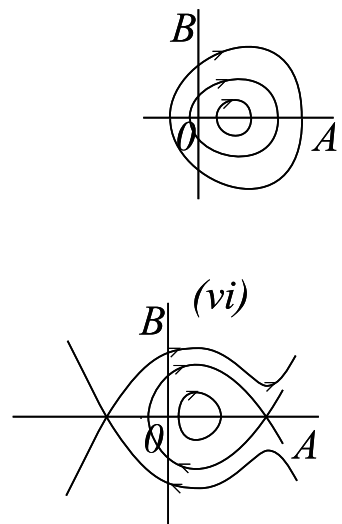

Figure 4: Phase portraits in the $(A, B)$ plane in the case $a=0 \quad(\beta=0)$ for system $(16,17,18)$. (i) $\nu>0, c<0, H=0$, (ii) $\nu>0, c<0,0<H<H_{m}$, (iii) $\nu>0, c<0, H=H_{m}$, (iv) $\nu>0, c<0, H_{m}<H$, (v) $\nu<0, c>0, H=0$, (vi) $\nu<0, c>0,0<H<H_{m}$. 